\title{
Mimicking Dermatopathic Lymphadenitis: An Entity of Lymphoma
}

\author{
Xiao-lin WU, Lan-ping DIAO, Zheng-xiang YANG, Ruijuan MA, Qingna GUO, Li-li WU \\ Hebei Medical University, Fourth Hospital, Department of Hematology, Shijiazhuang, Shijiazhuang, CHINA
}

To the Editor,

Dermatopathic lymphadenitis (DL) is described as a histologic entity which presents as a benign lymphatic hyperplasia. ${ }^{1}$ The histopathologic diagnosis of patients with enlarged lymph nodes classically includes a variety of exfoliative or eczematoid inflammatory erythrodermas. Some patients have been shown to have lymphomas. Because the management of DL differs from malignant lymphoproliferative disorders, the differential diagnosis should be emphasized. We present a case of a 14-year-old boy with of a right inguinal mass. The histopathologic diagnosis was a DL based on enlarged lymph nodes in the iliac region. Because the clinical status and imaging findings were not consistent with the histologic features of a DL, an ultrasound-guided core needle biopsy (CNB) of the right iliac mass was obtained. The final histopathologic diagnosis was defined as a T-cell lymphoma. The purpose of this report was to assist physicians in timely thought processes, the collection and analysis of clinical data, and avoiding or reducing diagnostic errors. The differential diagnosis of the case is presented.

\section{Case Report}

A 14-year-old boy presented to the Department of Hematology with a 1-year history of a slow-growing, right groin mass and a 2-week history of a painful sensation in the right lower extremity with a move- ment disorder. He had fever up to $40.2^{\circ} \mathrm{C}$ that started during the last 3 days with a productive cough. The patient has been taking antibiotics for an upper respiratory tract infection without any improvement. His personal history was unremarkable. The physical examination revealed an ill-defined, firm, severely painful enlarged and fleshy mass on the right-side of the groin, measuring $10-14 \mathrm{~cm}$ in diameter. Edema and a movement disorder of the right lower limb due to pain was noted, with no changes to the surrounding skin. Xerosis cutis was present on the palms of the hands and soles of the feet. No other abnormalities were detected. The patient had a computed tomography (CT) scan of the cervical neck, thorax, abdomen, and pelvis. The images demonstrated destruction of the right superior ramus of the pubis with a soft tissue neoplasm $(6 \times 9 \mathrm{~cm})$ surrounding the bony erosion, and multiple lymphadenectasis on the right side of the iliac blood vessels and inguinal region. Routing blood testing showed leukocytosis $\left(18.730 / \mathrm{mm}^{3}\right)$ with increased neutrophils $(86 \%)$ and an increased C-reactive protein level $(143 \mathrm{mg} / \mathrm{L})$ and erythrocyte sedimentation rate (ESR; $86 \mathrm{~mm} / 1 \mathrm{st} \mathrm{h}$ ). Liver and renal functions were normal, as were the immunoglobulin levels (IgA, IgG, and $\operatorname{IgM}$ ), lactate dehydrogenase (LDH) level, ANA spectrum, and urine analysis. In addition, specific serum tests for cytomegalovirus, Epstein-Bar virus, and human immunodeficiency virus were performed and shown to be negative. An interferon- $\gamma$-releasing test for tuberculosis was negative. 

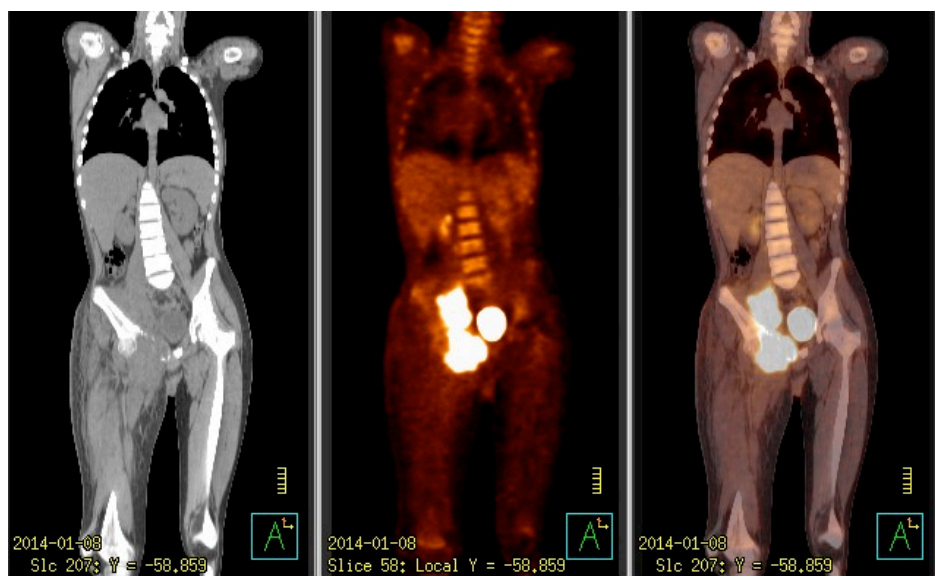

Figure 1. F-18 FDG PET/CT fusion images show intensely FDG-avid soft tissue mass of at the right-sided pelvic cavity and inguinal region (maximal standardized uptake value 21.9) with noticeable edema of the right lower limb.

A bone marrow smear had no apparent abnormalities. The results of the electrocardiogram and echocardiography were normal. Because of the high fever, an intravenous antibiotic regimen was initiated with a third-generation cephalosporin plus a $\beta$-lactamase inhibitor. Morphine and related analgesics were administered due to the intense right thigh pain. Despite treatment, the clinical status showed no improvement. Biopsies of the enlarged lymph nodes in the right inguinal region were obtained and a diagnosis of lymphoma was suggested. However, the results of the histologic examination of the lymph node specimens were diagnostic of lymph node reactive hyperplasia associated Tzone lymphocyte hyperplasia. Simultaneously, the cytoplasmic pigment was shown in the marginal zone of the lymph nodes. The pathologic sections were sent to a referral hospital in consultation. The histopathologic diagnosis was DL and lymphatic tissue reactive hyperplasia in the lymph nodes. An immunohistochemical study was positive for CD20 in the follicular zone, positive in the FDC reticulum for $\mathrm{CD} 21$, focal positive for $\mathrm{S}-100$, positive for CD1a, positive for $\mathrm{Ki}-67$ in $5-10 \%$, and negative for CD30.

DL has a benign clinical course and histopathologic findings. Macroscopically, the lymph nodes are moderately enlarged, firm, mobile, and painless. $^{2}$ The symptoms of DL alone are expected to be mild. ${ }^{1}$ The clinical presentation of DL is characterized by lymph node involvement instead of bony involvement and frequently includes a generalized skin disorder. ${ }^{3}$ These characteristics of DL were not consistent with the patient's clinical manifestations, such as immobile enlarged lymph nodes, severe pain, and destruction of bone. A pathologist, orthopedist, and hematologist were consulted, and the search for another source of the malignant neoplasm was added to the diagnostic process. One month after admission, the clinical symptoms of the patient were characterized by progressive growth of the tumor in the right inguinal region, severe pain in the right lower extremity, and a high fever. The patient underwent a F-18 FDG PET/CT scan. The F-18 FDG PET/CT imaging showed the following: 1. an intense FDG-avid soft tissue mass, $11 \mathrm{~cm} \times 7 \mathrm{~cm}$ in diameter, in the right pelvic cavity and inguinal region with a maximal standardized uptake value (SUV) of 21.9 and accompanied with osteolytic destruction of the adjacent bone; 2. a soft tissue nodule anterior to the right iliac vessels and anterior aspect of the sacrum with FDG-avid (maximal SUV=11.5); the erector spinae muscle at the fifth lumbar vertebral body had an abnormally intense high metabolic region (maximal SUV= 10.7); the FDG uptake of multiple enlarged lymph nodes in the right inguinal region was higher than normal; the cortical bone of the right femoral lesser trochanter was discontinuous with a hypodense fracture line in the medullary cavity, which did not show hypermetabolism; and the muscle tissue in the surrounding right femur was swollen and distended, and many small lymph nodes in the retroperitoneum were noted, although the lymph nodes 
did not show an abnormal high metabolic region with (18)F-FDG PET. Based on these features, a diagnosis of lymphoma was highly suspected. It was difficult to perform a biopsy again in the right inguinal region because a skin blister had developed due to severe right lower limb swelling. Thus, an ultrasound-guided core needle biopsy (CNB) was obtained from the mass in the right inguinal region. The neoplastic tissue specimens were sent for evaluation in a referral hospital. The histologic diagnosis was identified as an ALK+ anaplastic large cell lymphoma. The immunohistochemical staining results were as follows: negative for $\mathrm{CD} 2$, CD3, CD19, CD34, CD10, CD20, MPO, TdT, PGM1, CK, NSE, S-100, and lysozyme; and positive for CD99, CD117, CD30, ALK, EMA, CD68, and $\mathrm{Ki} 67$ in $90 \%$ of specimens. The final diagnosis in our patient was confirmed to be an ALK+ anaplastic large cell lymphoma, IV B. After undergoing chemotherapy with hyper-CAVD, the clinical features improved markedly. Currently, the patient is stable without any clinical signs of fever, osteodynia, swelling of the right lower limb, or lymphadenectasis.

\section{DISCUSSION}

DL is described histologically as enlarged lymph nodes, usually secondary to skin lesions, which are characterized by itching and scratching. ${ }^{2}$ Occasionally, DL has been reported in patients without active dermatopathies. ${ }^{3}$ Macroscopically, the enlarged lymph nodes are firm, moderately mobile, and rather painless. ${ }^{4}$ Histologically, the lesion is characterized by melanin and fat-laden proliferated reticular elements (histiocytes and macrophages) residing in the cortical regions of the lymph nodes. Currently, DL is thought to be a benign histopathologic finding. The clinical course of DL is also considered benign and lacks acute or severe symptoms. Usually, DL presents as a self-limiting process and requires no specific therapy. ${ }^{1} \mathrm{DL}$ is a rare disease; case series describing the cytologic and clinical features of DL are limited. Based on a review of the relevant literature, DL occasionally presents as a lymphoma. Aurer et al. ${ }^{4}$ reported that the pathologic diagnosis from a biopsy of a subcutaneous lymph node was DL, yet deeper nodes (the mediastinal and root of the mesentery) were diagnosed as Hodgkin's lymphoma in 2 of 10 cases reviewed. Aurer et al. ${ }^{4}$ emphasized that to make this diagnosis on a subcutaneous node does not necessarily preclude the existence of a lymphoblastoma. Cooper et al. ${ }^{5}$ reviewed 40 cases, and also found the co-existence of malignant lymphoma and DL in two patients. In addition to being complicated with two diseases, DL can be clinically similar to a lymphoma. ${ }^{6,7}$ and even exhibit the same imaging features. Makis et al. ${ }^{8}$ reported a patient with intensely FDG-avid lymph nodes in the cervical neck, axillary, and inguinal regions on F-18 FDG PET/CT imaging, which is commonly used in the evaluation of lymphomas; nevertheless, the histopathologic examination revealed a DL.

In this report the boy presented with worrisome symptoms of a large mass with intense pain and dyscinesia in the right lower extremity, a high fever, and failed antibiotic treatment. Imaging studies (CT scan and F-18 FDG PET/CT) yielded positive findings for neoplasia and bony destruction. The persistent severe clinical symptoms with the imaging findings were inconsistent with the pathologic diagnosis of DL; the treatment strategy and prognosis for patients with DL and malignant diseases differ. It was the underlying questions that prompted us to attempt a second biopsy with which to identify the diagnosis of a lymphoma. Moreover, the co-existence of lymphoma and DL could not be excluded in our case. Indeed, the histopathologic findings for the two diseases constitute the definitive diagnosis.

In conclusion, DL is a rare benign condition that can mimic a malignant disease. We re-emphasize the importance of the pathologic examination combined with the knowledge of clinical findings and imaging features.

\section{Grant sponsor:}

The foundation of Hebei province University's Advantage and Characteristic Discipline, China

\section{REFERENCES}

1. Psarommatis I, Vontas H, Gkoulioni V, et al. Dermatopathic lymphadenitis imitating a deep neck space infection. Am J Otolaryngol 30: 419-422, 2009. 
International Journal of Hematology and Oncology

2. Verma SK, Chowdhury N. A case of dermatopathic lymphadenitis diagnosed by fine needle aspiration. Pathology 38: 466468, 2006.

3. Winter LK, Spiegel JH, King T. Dermatopathic lymphadenitis of the head and neck. J Cutan Pathol 34: 195-197, 2007.

4. Aurer C, Grandbois J, Gaumond E. Dermatopathic lipomelanotic reticular cell hyperplasia of lymph nodes. Can Med Assoc J 66: 245-248, 1952

5. Cooper RA, Dawson PJ, Rambo ON. Dermatopathic lymphadenopathy: a clinico-pathologic analysis of lymph node biopsy over a fifteen-year period. Calif Med 106: 110$175,1967$.

6. Severson GS, Harrington DS, Burnett DA, et al. Dermatopathic lymphadenopathy associated with carbamazepine: a case mimicking a lymphoid malignancy. Am J Med 83: 597599, 1987.

7. Good DJ, Gascoyne RD. Atypical lymphoid hyperplasia mimicking lymphoma. Hematol Oncol Clin North Am 23: 729-745, 2009.

8. Makis W, Hickeson M, Blumenkrantz M. Interesting image Dermatopathic lymphadenitis: a pitfall for lymphoma evaluation by F-18 FDG PET/CT. Clin Nucl Med 35: 872-874, 2010.

\section{Correspondence:}

Li-li WU

Hebei Medical University

Fourth Hospital

Department of Hematology

050011 SHIJIAZHUANG / CHINA

Tel: $t 8631186096334$

Fax: t86 31186077634

e-mail: lily770215@163.com 